\title{
Existence Theorems for Nonlinear Evolution Inclusions $(*)$.
}

\author{
Tiziana Cardinali - Francesca Papaíini (**)
}

Summary. - In this paper we obtain an existence theorem for the abstract Cauchy problem for multivalued differential equations of the form $u^{\prime} \in-\partial^{-} f(u)+G(u), u(O)=x_{0}$, where $\partial^{-} f$ is the Fréchet subdifferential of a function $f$ defined on an open subset $\Omega$ of a real separable Hilbert space $H$, taking its values in $R \cup\{+\infty\}$ and $G$ is a multifunction from $C([0, T], \Omega)$ into the nonempty subsets of $L^{2}([0, T], H)$. As an application we obtain an existence theorem for the multivalued perturbed problem $x^{\prime} \in \partial^{-} f(x)+F(t, x), x(0)=x_{0}$, where $F:[0, T] \times \Omega \rightarrow n(H)$ is a multifunction satisfying some regularity assumptions.

\section{1. - Introduction.}

In the theory of the differential inclusions an important place is for non linear evolution equations, which, in the last few years, have been widely investigated by many Authors (cf. for istance, [7], [3], [9], [8], [5], [10], [4]). In 1989 M. TosquEs [10] has obtained an existence Theorem for the quasi-autonomous evolution problem of the form:

$$
\left\{\begin{array}{l}
x^{\prime} \in-\partial^{-} f(x)+h, \\
x(O)=x_{0}, \quad x_{0} \in D(f),
\end{array}\right.
$$

where $\partial^{-} f$ is the Fréchet subdifferential of a function $f$ defined on an open subset $\Omega$ of a real separable Hilbert space $H$, taking its values in $R \cup\{+\infty\}$ and having a $\varphi$-monotone subdifferential of order two, while $h \in L^{2}([O, T], H)$ is a singlevalued perturbation.

In order to obtain existence results for the Cauchy problem $(\mathrm{P})$, where the singlevalued perturbation is replaced with a multivalued one, in 1991 G. CoLomBo and M.

(*) Entrata in Redazione il 9 maggio 1994, in versione riveduta il 14 novembre 1995.

Indirizzo degli AA.: Department of Mathematies of Perugia University, Via Vanvitelli 1, Perugia, 06100, Italy.

(**) E-mail: papalini@guess.dipmat.unipg.it 
Tosques [4] have studied the abstract Cauchy problem

$$
\left\{\begin{array}{l}
u^{\prime} \in-\partial^{-} f(u)+G(u), \\
u(O)=x_{0}, \quad x_{0} \in D(f),
\end{array}\right.
$$

proving that (cf. [4], Theorem 3.1) it has solutions if $f: \Omega \rightarrow R \cup\{+\infty\}$ is a function with the properties:

i) $f$ has a $\varphi$-monotone subdifferential of order two;

ii) $\exists r^{\prime}>0$ such that $\mathrm{cl} B\left(x_{0}, r^{\prime}\right) \subset \Omega$ and the set

$$
L(c)=\left\{x \in \operatorname{cl} B\left(x_{0}, r^{\prime}\right): f(x) \leqslant c\right\} \quad \text { is compact in } H, \quad \forall c \in R,
$$

and moreover $G$, from $C([0, T], \Omega)$ into the nonempty, closed and convex subsets of $L^{2}([0, T], H)$, is a $\mathrm{H}$-u.s.c. and locally bounded multifunction.

In the first part of this note we have considered the abstract Cauchy problem (1) and we have obtained an existence result. We have proved that the problem (1) has solutions by assuming, besides the condition i), that $x_{0} \in D\left(\partial^{-} f\right)$, and

(ii) $\exists k, r>0:\left\|\operatorname{grad}^{-} f(x)\right\| \leqslant k, \forall x \in \operatorname{cl} B\left(x_{0}, r\right) \cap D\left(\partial^{-} f\right)$;

j) $G$ has sequentially closed graph in $C\left([0, T], H_{w}\right) \times L^{2}([0, T], H)_{w}$;

jj) $G(u)$ is closed and convex, $\forall u \in C([0, T], \Omega)$;

jjj) there exist $\gamma \in L^{2}\left([0, T], R^{+}\right)$and $\varepsilon>0$ such that $\|G(u)(t)\| \leqslant \gamma(t)$, a.e. in $[0, T], \forall u \in \operatorname{cl} B\left(u^{0}, \varepsilon\right)$, where $u^{0} \in C([0, T], \Omega)$ is the function defined by $u^{0}(t)=x_{0}, \forall t \in[0, T]$.

Our Theorem 1 extends the mentioned Theorem of G. Colombo - M. Tosques [4] in the sense that there exist functions $f: H \rightarrow R \cup\{+\infty\}$ satisfying the assumptions i), ii) of 'Theorem 1 but which do not satisfy the condition ii)' of Theorem of [4].

As an application of Theorem 1, in the second part of this note, we have proved the existence of solutions to multivalued perturbed problem

$$
\left\{\begin{array}{l}
x^{\prime} \in-\partial^{-} f(x)+F(t, x) \\
x(0)=x_{0}, \quad x_{0} \in D\left(\partial^{-} f\right)
\end{array}\right.
$$

where $f: \Omega \rightarrow R \cup\{+\infty\}$ satisfies, as in theorem 1, the conditions i), ii), while $F:[0, T] \times \Omega \rightarrow n(H)$ is a multifunction with the properties:

$\beta) F(t, x)$ is closed and convex, $\forall(t, x) \in[0, T] \times \Omega$;

$\beta \beta) F$ is measurable in $([0, T] \times \Omega, \mathfrak{L} \otimes \Re)$, where $\mathscr{L}$ is the Lebesgue $\sigma$-algebra of $[0, T]$ and $B$ indicates the family of the Borel subsets of $\Omega$;

$\beta \beta \beta)$ for a.e. $t \in[0, T], x F(t, x)$ has sequentially closed graph in $H_{w} \times H_{w}$; 
$\beta \mathrm{v})$ there exists $\gamma \in L^{2}\left([0, T], R^{+}\right)$such that $\|F(t, x)\| \leqslant \gamma(t)$, a.e. in $[0, T]$, $\forall x \in \Omega$.

\section{2. - Preliminaries.}

Let $\mathrm{E}$ and $\mathrm{F}$ be two Hausdorff topological vector spaces and $N_{E}(0), N_{F}(0)$ be two bases of balanced and absorbent neighborhoods of zero in $E$ and in $F$ respectively.

Given a subset $X$ of $E$ and a subset $Y$ of $F$, we denote with $n(Y)$ the family of nonempty subsets of $Y$. A multifunction $\Gamma: X \rightarrow n(Y)$ is called Hausdorff upper semicontinuous ( $H$-u.s.c.) in $X$ if

(H-u.s.c.) $\forall x \in X$ and $\forall V \in N_{F}(0)$ there exists $U \in N_{E}(0)$ with the property $\Gamma[(x+U) \cap X] \subset \Gamma(x)+V$

while $\Gamma$ is said to have closed graph if the set $\operatorname{gr}(\Gamma)=\{(x, y) \in X \times Y: y \in \Gamma(x)\}$ is closed in $E \times F$.

Let now $(Z, \mathfrak{A})$ be a measurable space and $Y$ be a metric space. A multifunction $\Gamma: Z \rightarrow n(Y)$ is said to be measurable if for any open subset $A \subset Y$, the set

$$
\Gamma^{-}(A)=\{x \in Z: F(x) \cap A \neq \emptyset\}
$$

belongs to $\mathfrak{a}$.

In what follows, $H$ is a real separable Hilbert space, with norm $\|\cdot\|$ endowed by the scalar product $\langle.,$.$\rangle and we denote by H_{w}$ the space $H$ with the weak topology. For $x \in H$ and $\varepsilon>0$ we set $B(x, \varepsilon)=\{y \in H:\|y-x\|<\varepsilon\}, \operatorname{cl} B(x, \varepsilon)=\{y \in H:\|y-x\| \leqslant$ $\leqslant \varepsilon\}$ represents the clousure of $B(x, \varepsilon)$; moreover, if $A$ is a subset of $H$, we put $B(A, \varepsilon)=\{x \in H: d(x, A)<\varepsilon\}$, where

$$
d(x, A)=\inf \{\|y-x\|: y \in A\} \quad \text { and } \quad\|A\|=\sup \{\|y\|: y \in A\} .
$$

Given a positive number $T$, we indicate the norm of the space $L^{2}([0, T], H)$ by $\|\cdot\|_{2}$ and we denote by $L^{2}([0, T], H)_{W}$ the space $L^{2}([0, T], H)$ where we consider the weak topology.

Given an open subset $\Omega$ of $H$ and a function $f: \Omega \rightarrow R \cup\{+\infty\}$, the multifunction a- $f: \Omega \rightarrow 2^{H}$, defined as follows

$$
\partial^{-} f(x)= \begin{cases}\emptyset, & \text { if } f(x)=+\infty, \\ \left\{\alpha \in H: \liminf _{y \rightarrow x} \frac{f(y)-f(x)-\langle\alpha, y-x\rangle}{\|y-x\|} \geqslant 0\right\}, & \text { if } f(x)<+\infty,\end{cases}
$$

is called the Fréchet subdifferential of $f$. The sets $D(f)=\{x \in \Omega: f(x)<+\infty\}$ and $D\left(\partial^{-} f\right)=\left\{x \in \Omega: \partial^{-} f(x) \neq \emptyset\right\}$ are the domains of $f$ and $\partial^{-} f$ respectively. 
Remark 1. - The values of $\partial^{-} f$ fare closed and convex (cf. [5], p. 1403).

For every $x \in D\left(\partial^{-} f\right)$ we denote by $\operatorname{grad}^{-} f(x)$ the element of minimal norm of $\partial^{-} f(x)$.

If $f: \Omega \rightarrow R \cup\{+\infty\}$ is a lower semicontinuous function, we say thatf has a $\varphi$-monotone subdifferential of order two if there exists a continuous map $\varphi:[D(f)]^{2} \times R^{2} \rightarrow R^{+}$such that

(2.1) for every $x, y \in D\left(\partial^{-} f\right)$ and for every $\alpha \in \partial^{-} f(x)$ and $\beta \in \partial^{-} f(y)$, we have $\langle\alpha-\beta, x-y\rangle \geqslant-\varphi(x, y, f(x), f(y))\left(1+\|\alpha\|^{2}+\|\beta\|^{2}\right)\|x-y\|^{2}$.

In the followings, if $T$ is a positive number and $\left.\left.T_{0} \in\right] 0, T\right]$, we adopt the convention: a function $h:\left[0, T_{0}\right] \rightarrow \Omega$ is automatically extended to $[0, T]$ by putting $h(t)=$ $\left.\left.=h\left(T_{0}\right), \forall t \in\right] T_{0}, T\right]$. Therefore, every multifunction $G$ from $C([0, T], \Omega)$ into the subsets of $L^{2}([0, T], H)$ induces a multifunction from $C\left(\left[0, T_{0}\right], \Omega\right)$ into the subsets of $L^{2}\left(\left[0, T_{0}\right], H\right)$ which we still denote by $G$.

The following Proposition 1 is a version of the Theorem 3.6 of M. Tosques (ef.[10], p. 82).

PRoposition 1 (cf.[10]). - Let $f: \Omega \rightarrow R \cup\{+\infty\}$ be a function with a $\varphi$-monotone subdifferential of order two. Then

$\forall x_{0} \in D(f), \forall M \geqslant 0$ such that $f\left(x_{0}\right) \leqslant M, \exists T^{*}>0$ with the property:

$\forall T>0$ and $\forall h \in L^{2}([0, T], H)$ with $\|h\|_{2} \leqslant M$, there exists a unique function $u_{h}:\left[0, T_{0}\right] \rightarrow \Omega$, where $T_{0}=\min \left\{T^{*}, T\right\}$, that is a solution of the Cauchy problem:

$(\mathrm{P})_{h}$

$$
\left\{\begin{array}{l}
x^{\prime} \in-\partial^{-} f(x)+h \\
x(0)=x_{0}
\end{array}\right.
$$

with the properties:

I) $u_{h}$ is continuous on $\left[0, T_{0}\right]$ and absolutely continuous on the compact subsets of $] 0, T_{0}[$;

II) $u_{h}(t) \in D\left(\partial^{-} f\right)$ and $u_{h}^{\prime}(t) \in-\partial^{-} f\left(u_{h}(t)\right)+h(t)$ a.e. in $\left[0, T_{0}\right]$;

III) $u_{h}(0)=x_{0}$;

IV) $u_{h}^{\prime} \in L^{2}\left(\left[0, T_{0}\right], H\right)$;

V) $\int_{0}^{t}\left\|u_{h}^{\prime}(s)\right\|^{2} d s \leqslant 2\left(f\left(x_{0}\right)-f\left(u_{h}(t)\right)\right)+\int_{0}^{t}\|h(s)\|^{2} d s, \forall t \in\left[0, T_{0}\right]$;

VI) $f \circ u_{h}$ is absolutely continuous on $\left[0, T_{0}\right]$;

VII) $\left(f \circ u_{h}\right)^{\prime}(t)=\left\langle h(t)-u_{h}^{\prime}(t), u_{h}^{\prime}(t)\right\rangle$ a.e. in $\left[0, T_{0}\right]$. 


\section{3. - Existence theorem for an abstract Cauchy problem.}

We consider the abstract Cauchy problem

$$
\left\{\begin{array}{l}
u^{\prime} \in-\partial^{-} f(u)+G(u) \\
u(0)=x_{0}
\end{array}\right.
$$

where $f: \Omega \rightarrow R \cup\{+\infty\}$ is a function with a $\varphi$-monotone subdifferential of order two and $G$ is a multifunction from $C([0, T], \Omega)$ into the nonempty subsets of $L^{2}([0, T], H)$.

A function $u:\left[0, T_{0}\right] \rightarrow \Omega$, where $\left.\left.T_{0} \in\right] 0, T\right]$, is called solution of Cauchy problem (1) if there exists $h \in G(u)$ such that:

a) $u$ is continuous on $\left[0, T_{0}\right]$ and absolutely continuous on the compact subsets of $] 0, T_{0}[$;

b) $u(t) \in D\left(\partial^{-} f\right)$ and $u^{\prime}(t) \in-\partial^{-} f(u(t))+h(t)$ a.e. in $\left[0, T_{0}\right]$;

c) $u(0)=x_{0}$.

In this section we present an existence theorem for the Cauchy problem (1).

THEOREM 1. - Let $\Omega$ be an open subset of a separable real Hilbert space $H$ and let $T$ be a positive number. Let $f: \Omega \rightarrow R \cup\{+\infty\}$ be a function, $G: C([0, T], \Omega) \rightarrow n\left(L^{2}([0, T], H)\right)$ be a multifunction and $x_{0} \in D\left(\partial^{-} f\right)$ such that:

i) $f$ has a $\varphi$-monotone subdifferential of order two;

ii) $\exists k, r>0:\left\|\operatorname{grad}^{-} f(x)\right\| \leqslant k, \forall x \in \operatorname{cl} B\left(x_{0}, r\right) \cap D\left(\partial^{-} f\right)$;

j) $G$ has sequentially closed graph in $C\left([0, T], H_{w}\right) \times L^{2}([0, T], H)_{w}$;

jj) $G(u)$ is closed and convex, $\forall u \in C([0, T], \Omega)$;

jjj) there exist $\gamma \in L^{2}\left([0, T], R^{+}\right)$and $\varepsilon>0$ such that $\|G(u)(t)\| \leqslant \gamma(t)$, a.e. in $[0, T], \forall u \in \operatorname{cl} B\left(u^{0}, \varepsilon\right)$ where $u^{0} \in C([0, T], \Omega)$ is the function defined by $u^{0}(t)=x_{0}$, $\forall t \in[0, T]$.

In these conditions, there exist a number $\left.\left.T_{0} \in\right] 0, T\right]$ and a function $u:\left[0, T_{0}\right] \rightarrow \Omega$ that is solution of the Cauchy problem (1).

Moreover u satisfies the following properties:

a) $u^{\prime} \in L^{2}\left(\left[0, T_{0}\right], H\right)$;

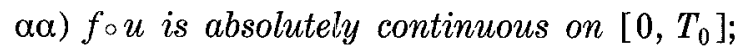

$\alpha \alpha \alpha) \int_{0}^{t}\left\|u^{\prime}(s)\right\|^{2} d s \leqslant 2\left(f\left(x_{0}\right)-f(u(t))\right)+\int_{0}^{t}\|G(u)(s)\|^{2} d s, \forall t \in\left[0, T_{0}\right]$. 
Proof. - First, we observe that from our hypotheses it is possible to find three positive numbers $R, M$ and $L$ such that:

$$
\begin{aligned}
& f(x) \geqslant f\left(x_{0}\right)-1, \quad \forall x \in \operatorname{cl} B\left(x_{0}, R\right), \\
& f\left(x_{0}\right) \leqslant M, \quad\|\gamma\|_{2} \leqslant M, \\
& \|G(u)(t)\| \leqslant \gamma(t), \quad \forall u \in C\left([0, T], \operatorname{cl} B\left(x_{0}, R\right)\right), \quad \text { a.e. in }[0, T], \\
& L=\sup \left\{\varphi\left(x_{1}, x_{2}, y_{1}, y_{2}\right): x_{1}, x_{2} \in \operatorname{cl} B\left(x_{0}, R\right) \cap D(f), y_{1}, y_{2} \Subset\right. \\
& \left.\in\left[f\left(x_{0}\right)-1, f\left(x_{0}\right)+\frac{1}{2} M^{2}\right]\right\}, \\
& \left\|\operatorname{grad}^{-} f(x)\right\| \leqslant k, \quad \forall x \in \operatorname{cl} B\left(x_{0}, R\right) \cap D\left(\partial^{-} f\right),
\end{aligned}
$$

where $\varphi:[D(f)]^{2} \times R^{2} \rightarrow R^{+}$is the continuous map that satisfies (2.1).

Choosen $T^{\prime}$ such that $0<T^{\prime}<R^{2} /\left(2+M^{2}\right)$, if $T^{*}$ is the positive number taken in correspondence of $f, x_{0}$ and $M$ as in the Proposition 1 (cf. (3.2)), let $T_{0}=$ $=\min \left\{T^{\prime}, T^{*}, T\right\}$.

Put $\mathfrak{V}=\left\{h \in L^{2}\left(\left[0, T_{0}\right], H\right):\|h(t)\| \leqslant \gamma(t)\right.$, a.e. in $\left.\left[0, T_{0}\right]\right\}$ and let $s: \mathcal{V} \rightarrow C\left(\left[0, T_{0}\right], \Omega\right)$ be the function defined by $s(h)=u_{h}$, where $u_{h}$ is the unique solution of the problem $(\mathrm{P})_{h}$ (cf. Proposition 1).

First we claim that, for every $h \in \mathfrak{V}$, we have

$$
\left\|u_{h}(t)-x_{0}\right\| \leqslant R, \quad \forall t \in\left[0, T_{0}\right] .
$$

Indeed, from V) of the Proposition 1 it follows

$$
\begin{aligned}
\left\|u_{h}(t)-x_{0}\right\| \leqslant \int_{0}^{t}\left\|u_{h}^{\prime}(s)\right\| d s \leqslant \sqrt{t}\left(\int_{0}^{t}\left\|u_{h}^{\prime}(s)\right\|^{2} d s\right)^{1 / 2} & \leqslant \\
& \leqslant \sqrt{t}\left[2\left(f\left(x_{0}\right)-f\left(u_{h}(t)\right)\right)+M^{2}\right]^{1 / 2} .
\end{aligned}
$$

Let $\bar{T}=\sup \left\{t \in\left[0, T_{0}\right]:\left\|u_{h}(s)-x_{0}\right\| \leqslant R, \forall s \in[0, t]\right\}$; by the continuity of $u_{h}$ we have that $\bar{T}>0$. Therefore, to obtain (3.6) it is sufficient to prove that $\bar{T}=T_{0}$. Indeed, if $\bar{T}<T_{0}$ by (3.7), (3.1) and by recalling that $T^{\prime}<R^{2} /\left(2+M^{2}\right)$, it follows that $\left\|u_{h}(\bar{T})-x_{0}\right\|<\sqrt{T^{\prime}}\left[2+M^{2}\right]^{1 / 2} \leqslant R$, but this inequality, being $u_{h}$ continuous on $\left[0, T_{0}\right]$, contradicts the definition of $\bar{T}$. So (3.6) holds. Let

$$
\begin{aligned}
\mathcal{W}=\left\{u \in C\left(\left[0, T_{0}\right], \Omega\right): u(t)=x_{0}+\int_{0}^{t} \omega(s) d s,\right. & \\
& \left.\forall t \in\left[0, T_{0}\right],\|\omega(t)\| \leqslant k+\gamma(t), \quad \text { a.e. in }\left[0, T_{0}\right]\right\} .
\end{aligned}
$$

It is easy to see that $w$ is strongly equicontinuous and so it is equicontinuous in 
$\left.C\left(0, T_{0}\right], H_{w}\right)$; moreover, for every $t \in\left[0, T_{0}\right]$, the set $W(t)=\{u(t) \in H: u \in \mathfrak{W}\}$ is relatively compact in $H_{w}$, therefore, from Arzelà-Ascoli Theorem, it follows that $W$ is relatively compact in $C\left(\left[0, T_{0}\right], H_{w}\right)$. Now we claim that wo is closed in $C\left(\left[0, T_{0}\right], H_{w}\right)$ too. Let $\left(u_{\alpha}\right)_{\alpha}$ be a net in $w, u_{\alpha} \rightarrow u$ in $C\left(\left[0, T_{0}\right], H_{w}\right)$. Then $u_{\alpha}(t)=x_{0}+\int_{0}^{t} \omega_{\alpha}(s) d s$, $\forall t \in\left[0, T_{0}\right]$, and $\left\|\omega_{\alpha}(t)\right\| \leqslant k+\gamma(t)$, a.e in $\left[0, T_{0}\right]$. By passing to a subnet, if necessary, we may assume that $\omega_{a} \rightarrow \omega$ in $L^{2}([0, T], H)_{w}$. Therefore we obtain that, $\forall t \in\left[0, T_{0}\right]$, $u_{\alpha}(t) \rightarrow x_{0}+\int_{0}^{t} \omega(s) d s$ in $H_{w}$, which implies that $u(t)=x_{0}+\int_{0}^{t} \omega(s) d s, \forall t \in\left[0, T_{0}\right]$, and, of course, $\|\omega(t)\| \leqslant k+\gamma(t)$, a.e in $\left[0, T_{0}\right]$. Hence $w$ is a compact subset of $C\left(\left[0, T_{0}\right], H_{w}\right)$.

Now, from the last statement of the proof of Lemma 3.21 of [10] and from the property VII) of the Proposition $1, \forall h \in \mathcal{V}$ we have that

$$
\left\|u_{h}^{\prime}(t)\right\| \leqslant\left\|\operatorname{grad}^{-} f\left(u_{h}(t)\right)\right\|+\|h(t)\|, \quad \text { a.e. in }\left[0, T_{0}\right],
$$

therefore, by (3.6) and our assumptions, it follows that

$$
\left\|u_{h}^{\prime}(t)\right\| \leqslant k+\gamma(t), \quad \text { a.e. in }\left[0, T_{0}\right] .
$$

Then, taking the properties I) and IV) of Proposition 1 into account, $s(\mathfrak{V})$ is a subset of w.

Now we want to show that function $s$ is continuous from $v$ with the topology of $L^{2}\left(\left[0, T_{0}\right], H\right)_{w}$ into $W$ with the topology of $C\left(\left[0, T_{0}\right], H_{w}\right)$. First, we observe that the space $W$ with the topology of $C\left(\left[0, T_{0}\right], H_{w}\right)$ is meterizable because this topology in w is equivalent to the topology of pointwise convergence on a countable dense subset of $\left[0, T_{0}\right]$; so, since $w$ is compact in the space $C\left(\left[0, T_{0}\right], H_{w}\right)$, it is sufficient to prove that $\operatorname{gr}(s)$ is sequentially closed in $v \times w$. Then let $\left(h_{n}, u_{n}\right) \in g r(s),\left(h_{n}, u_{n}\right) \rightarrow(\widehat{h}, u)$ in $L^{2}\left(\left[0, T_{0}\right], H\right)_{w} \times C\left(\left[0, T_{0}\right], H_{w}\right)$ and let $\widehat{u}=s(\widehat{h})$. By (3.6), (3.1) and by the property $\mathrm{V})$, we obtain

$$
f\left(u_{n}(t)\right) \in\left[f\left(x_{0}\right)-1, f\left(x_{0}\right)+\frac{1}{2} M^{2}\right], \quad \forall n \in N, \quad \forall t \in\left[0, T_{0}\right] .
$$

Moreover, since $f$ has a $\varphi$-monotone subdifferential of order two, by (3.6), (3.9), (3.4) and (3.8), we have

$$
\begin{aligned}
& \left\langle u_{m}^{\prime}(t)-\widehat{u}^{\prime}(t), u_{m}(t)-\widehat{u}(t)\right\rangle \leqslant\left\langle h_{m}(t)-\widehat{h}(t), u_{m}(t)-\widehat{u}(t)\right\rangle+ \\
& +L\left(1+\left\|h_{m}(t)-u_{m}^{\prime}(t)\right\|^{2}+\left\|\widehat{h}(t)-\widehat{u}^{\prime}(t)\right\|^{2}\right)\left\|u_{m}(t)-\widehat{u}(t)\right\|^{2} \leqslant \\
& \leqslant\left\langle h_{m}(t)-\widehat{h}(t), u_{m}(t)-\widehat{u}(t)\right\rangle+\delta(t)\left\|u_{m}(t)-\widehat{u}(t)\right\|^{2}, \\
& \quad \forall m \in N, \quad \text { for a.e. } \mathrm{t} \in\left[0, \mathrm{~T}_{0}\right],
\end{aligned}
$$


where $\delta(t)=L\left[1+8(\gamma(t))^{2}+8 k \gamma(t)+4 k^{2}\right], \forall t \in\left[0, T_{0}\right]$; by integrating we obtain

$$
\begin{aligned}
& \frac{1}{2}\left\|u_{m}(t)-\widehat{u}(t)\right\|^{2} \leqslant \int_{0}^{t}\left\langle h_{m}(s)-\widehat{h}(s), u_{m}(s)-\widehat{u}(s)\right\rangle d s+ \\
& \quad+\int_{0}^{t} \delta(s)\left\|u_{m}(s)-\widehat{u}(s)\right\|^{2} d s, \quad \forall t \in\left[0, T_{0}\right], \quad \forall m \in N .
\end{aligned}
$$

Applying Gronwall's inequality (cf.[6], p. 36) we get

$$
\begin{aligned}
\left\|u_{m}(t)-\widehat{u}(t)\right\|^{2} \leqslant & \alpha_{m}(t)+ \\
& +\int_{0}^{t} 2 \delta(s)\left|\alpha_{m}(s)\right| \exp \left(\int_{0}^{s} 2 \delta(v) d v\right) d s, \quad \forall t \in\left[0, T_{0}\right], \quad \forall m \in N,
\end{aligned}
$$

where $\alpha_{m}(t)=2 \int_{0}^{t}\left\langle h_{m}(s)-\widehat{h}(s), u_{m}(s)-\widehat{u}(s)\right\rangle d s$.

Since (cf. [2], Prop. III.5)

$$
\lim _{m \rightarrow \infty} \alpha_{m}(t)=0, \quad \forall t \in\left[0, T_{0}\right],
$$

and

$$
\left|\alpha_{m}(t)\right| \leqslant 8\left(\left\|x_{0}\right\|+R\right)\|\gamma\|_{1}, \quad \forall t \in\left[0, T_{0}\right] \quad \text { and } \quad \forall m \in N,
$$

we have

$$
\lim _{m \rightarrow \infty}\left\|u_{m b}(t)-\widehat{u}(t)\right\|^{2}=0, \quad \forall t \in\left[0, T_{0}\right],
$$

that is $u_{m} \rightarrow \widehat{u}$ in $C\left(\left[0, T_{0}\right], \Omega\right)$ and so $\widehat{u}=u$; therefore $\operatorname{gr}(s)$ is sequentially closed in $\mathfrak{v} \times \mathfrak{w}$.

Now, let $R:\urcorner \rightarrow n(\mathfrak{\vartheta})$ be the multifunction defined by (cf. (3.3))

$$
R(h)=G(s(h)), \quad h \in \mathcal{V} .
$$

It is easy to see that $\operatorname{gr}(R)$ is sequentially closed in $L^{2}\left(\left[0, T_{0}\right], H\right)_{w} \times L^{2}\left(\left[0, T_{0}\right], H\right)_{w}$; therefore, since $V$ is a compact and meterizable subset of space $L^{2}\left(\left[0, T_{0}\right] H\right)_{w}$, we have that $R$ is $H$-u.s.c. Then, by Fan-Glicksberg fixed point Theorem,the multifunction $R$ has a fixed point, that is a solution of the Cauchy problem (1) satisfying the conditions $\alpha$ ), $\alpha \alpha), \alpha \alpha \alpha)$.

REMARK 2. - Observe that the previous result extends Theorem 3.1 of [4], in the sense that there exist functions $f: H \rightarrow R \cup\{+\infty\}$ satisfying the assumptions i) and ii) of theorem 1 but which do not satisfy the conditions of theorem 3.1 of [4] as it fol- 
lows taking the function $f: l^{2} \rightarrow R \cup\{+\infty\}$ defined by

$$
f(x)=\|x\|, \quad \forall x \in l^{2},
$$

into account.

\section{4. - An existence theorem for perturbed Cauchy problem.}

In this section we consider the multivalued perturbed problem:

$$
\left\{\begin{array}{l}
x^{\prime} \in-\partial^{-} f(x)+F(t, x) \\
x(0)=x_{0}
\end{array}\right.
$$

where $F:[0, T] \times \Omega \rightarrow n(H)$ is a multifunction.

A function $u:\left[0, T_{0}\right] \rightarrow \Omega$, where $\left.\left.T_{0} \in\right] 0, T\right]$, is called solution of Cauchy problem (2) if there exists a function $h \in L^{2}\left(\left[0, T_{0}\right], H\right), h(t) \in F(t, u(t))$ a.e. in $\left[0, T_{0}\right]$, such that:

a) $u$ is continuous on $\left[0, T_{0}\right]$ and absolutely continuous on the compact subsets of $] 0, T_{0}[$;

b) $u(t) \in D\left(\partial^{-} f\right)$ and $u^{\prime}(t) \in-\partial^{-} f(u(t))+h(t)$ a.e. in $\left[0, T_{0}\right]$;

c) $u(0)=x_{0}$. by

Let $G: C([0, T], \Omega) \rightarrow 2^{L^{2}([0, T], H)}$ be the multivalued Nemytski operator defined

$$
G(u)=\left\{h \in L^{2}([0, T], H): h(t) \in F(t, u(t)), \text { a.e. in }[0, T]\right\},
$$

$$
\forall u \in C\left(\left[0, T^{\top}\right], \Omega\right)
$$

We state now some properties on the multifunction defined in (4.1) that allow us to obtain an existence result for the problem (2).

Lemma. - Let $F:[0, T] \times \Omega \rightarrow n(H)$ be a multifunction with the properties:

B) $F(t, x)$ is closed and convex, $\forall(t, x) \in[0, T] \times \Omega$;

$\beta \beta) F$ is measurable in $([0, T] \times \Omega, \mathfrak{L} \otimes \mathscr{B})$, where $\mathfrak{L}$ is the Lebesgue $\sigma$-algebra of $[0, T]$ and $\mathscr{B}$ indicates the family of the Borel subsets of $\Omega$;

$\beta \beta \beta)$ for a.e. $t \in[0, T], x F(t, x)$ has sequentially closed graph in $H_{w} \times H_{w}$;

$\beta v)$ there exists $\gamma \in L^{2}\left([0, T], R^{+}\right)$such that $\|F(t, x)\| \leqslant \gamma(t)$, a.e. in $[0, T]$, $\forall x \in \Omega$. 
In these conditions, the multifunction $G: C([0, T], \Omega) \rightarrow 2^{L^{2}([0, T], H)}$ defined in (4.1) has nonempty values and it satisfies the conditions $\mathrm{j}$ ), jj) and jjj) of Theorem 1.

Proof. - We start by observing that $G(u)$ is non empty and closed, $\forall u \in C([0, T], \Omega)$ (cf. Kuratowski and Ryll-Nardzewski Theorem); moreover it is easy to see that $G(u)$ is convex, $\forall u \in C([0, T], \Omega)$, and that $G$ satisfies (cf. $\beta v)$ ) the assumption jjj).

Now we prove that $G$ has sequentially closed graph in $C\left([0, T], H_{w}\right) \times$ $\times L^{2}([0, T], H)_{w}$. Indeed, let $\left(u_{n}, h_{n}\right) \in \operatorname{gr}(G),\left(u_{n}, h_{n}\right) \rightarrow(u, h)$ in $C\left([0, T], H_{w}\right) \times$ $\times L^{2}([0, T], H)_{w}$. Since in a Banach space the strong closure of a convex subset coincides with the weak clousure and since $\left(h_{n}\right)_{n}$ converges weakly to $h$ in $L^{2}([0, T], H)$, for every $m \in N, h$ belongs to the strong clousure of $\operatorname{co}\left\{h_{n}\right\}_{n \geqslant m}$. Therefore, for every $m \in N$, there exists $v_{m} \in \operatorname{co}\left\{h_{n}\right\}_{n \geqslant m}, v_{m}=\sum_{n=m}^{\infty} \alpha_{n}(m) h_{n}$, where $a_{n}(m)=0$, except for a finite number of n's in which $a_{n}(m)>0$, and $\sum_{n=m}^{\infty} a_{n}(m)=1$, such that the sequence $\left(v_{m}\right)_{m}$ converges strongly in $L^{2}([0, T], H)$ to $h$. Then it is possible to find a subsequence of $\left(v_{m}\right)_{m}$, still denoted by $\left(v_{m}\right)_{m}$, such that $\left(v_{m}(t)\right)_{m}$ converges to $h(t)$, a.e. in $[0, T]$. By $\beta \beta \beta)$ and $\beta v$ ), for a.e. $t \in[0, T]$, the multifunction $x F(t, x)$ is upper semicontinuous with respect to the weak topology of $H$. Moreover, since $\left(u_{n}\right)_{n}$ converges to $u$ in $C\left([0, T], H_{w}\right)$, for a.e. $t \in[0, T]$, it follows that for every convex neighborhood $U$ of zero in $H_{w}$, there exists $n^{*} \in N$ such that

$$
F\left(t, u_{n}(t)\right) \subset F(t, u(t))+U, \quad \forall n \geqslant n^{*} ;
$$

hence

$$
h_{n}(t) \in F(t, u(t))+U, \quad \forall n \geqslant n^{*} .
$$

By multiplying the above inclusion by $a_{n}(m)$ and summing them, we deduce that

$$
v_{m}(t) \in F(t, u(t))+U, \quad \forall m \geqslant n^{*} .
$$

Then

$$
h(t) \in F(t, u(t))+c l U
$$

and, since $U$ is arbitrary, it follows that $h(t) \in F(t, u(t))$ a.e. in $[0, T]$, that is $h \in G(u)$.

From the above Lemma and from Theorem 1 we have the following existence result

Theorem 2. - Let $H, \Omega, T, f$ and $x_{0}$ be as in Theorem 1 and let $F:[0, T] \times \Omega \rightarrow n(H)$ be a multifunction with the properties $\beta$ ), $\beta \beta$ ), $\beta \beta \beta$ ) and $\beta \mathrm{v}$ ).

In these conditions, there exist a number $\left.\left.T_{0} \in\right] 0, T\right]$ and a function $u:\left[0, T_{0}\right] \rightarrow \Omega$ that is solution of the Cauchy problem (2). 
Moreover, $u$ also satisfies the following properties:

a) $u^{\prime} \in L^{2}\left(\left[0, T_{0}\right], H\right)$;

$\alpha \alpha) f \circ u$ is absolutely continuous on $\left[0, T_{0}\right]$;

$\alpha \alpha \alpha) \int_{0}^{t}\left\|u^{\prime}(s)\right\|^{2} d s \leqslant 2\left(f\left(x_{0}\right)-f(u(t))\right)+\int_{0}^{t}\|F(t, u(s))\|^{2} d s, \forall t \in\left[0, T_{0}\right]$.

\section{REFERENCES}

[1] J. P. Aubin - A. Cellina, Differential Inclusions, Springer-Verlag, Berlin (1984).

[2] H. BREZIS, Analyse fonctionnelle, théorie et applications, Masson, Paris (1983).

[3] A. Cellina - V. StaICU, On evolution equations having monotonicities of opposite sign, J. Differ. Equat., 90 (1991), pp. 71-80.

[4] G. CoLombo - M. TOSQUES, Multivalued perturbations for a class of nonlinear evolution equations, Ann. Mat. Pura Appl., 160 (1991), pp. 147-162.

[5] M. Degiovanni - A. Marino - M. Tosques, Evolution equations with lack of convexity, J. Nonlinear Anal. Th. Math. Appl., 9 (1985), pp. 1401-1443.

[6] J. Hale, Ordinary Differential Equations, Wiley-Interscience, New York (1969).

[7] D. Kravvaritis - N. S. Papageorgiou, Multivalued perturbations of subdifferential type evolution equations in Hilbert spaces, J. Differential Equations, 76 (1988), pp. 238-255.

[8] F. Papalini - S. Vercillo, Existence of solutions to a class of evolution equations, Riv. Mat. Univ. Parma (5), 3 (1994), pp. 209-220.

[9] V. Staicu, On the Existence of Solutions to a class of Differential Inclusions, Rend. Sem. Mat. Univ. Pol. Torino, Vol. 48, 2 (1990), pp. 137-148.

[10] M. TOSQUES, Quasi-autonomous parabolic evolution equations associated with a class of non linear operators, Ricerche di Matematica, 38 (1989), pp. 63-92. 